\title{
Signatura rerum: Semiotics of the Subnuclear
}

\author{
Arpita Roy, Max Planck Institute
}

\begin{abstract}
On July 4, 2012, experimental physicists on the Large Hadron Collider near Geneva, in Switzerland, announced the historic discovery of the Higgs boson. After analyzing trillions of proton-to-proton collisions, two teams of physicists concluded that signatures of diphotons in the final state were evidence of the long-sought-after Higgs particle. The answerability of a particle search to polysemic signals raises a deeply provocative question: In what ways does a sign facilitate the discovery of a thing? Drawing on fieldwork at the Large Hadron Collider complex, this article attempts to probe the semiosis of signatures as a palimpsest of inherent possibilities spanning the width of the universe, which is neither a contingent ordering of the Saussurean kind nor a brick-by-brick Peircean construct. What it eloquently foregrounds is the capacity of a class of signs to reflect the presence of objects, even those that are materially nonexistent, which resolves many a metaphysical perplexity involving language, thought, and reality. In the process, this article makes a case for the role of meaningful, qualitative evaluations, which lie at the root of experimental searches, and explains how these qualitative evaluations are a more trustworthy source of discoveries in particle physics than metrics or magnitudes.
\end{abstract}

You would not seek me, if you had not found me.

-Pascal (on God)

W hat is the context in which discoveries take place? Or could one say that discoveries take place precisely when they break free from their context? In the discussion that follows, I wish to focus on the semiosis of material discoveries to highlight the puzzle of how particle physicists

Contact Arpita Roy at MPI zur Erforshung multireligioser und multiethnischer, Hermann-Föge-Weg 11, 37073 Göttingen, Niedersachsen, Germany (roy@mmg.mpg.de).

I would like to thank a few physicists from CERN involved with this research: Michael Doser, for the thoughtfulness and extraordinary cogency with which he explained every aspect of the experiments; Luis

Signs and Society, vol. 5, no. S1 (Supplement 2017). ๑ 2017 Semiosis Research Center at Hankuk University of Foreign Studies. All rights reserved. 2326-4489/2017/05S1-0004\$10.00 
strive to recognize new particles by means of a class of signs called "signatures." In analyzing this problem of how materiality is approached through the intermediary of signs, I will turn to the recent discovery of the Higgs particle from experiments conducted on the world's largest accelerator, the Large Hadron Collider (LHC), near Geneva, in Switzerland. Built at a staggering cost of 3.5 billion Swiss francs, spread over fifteen years, the LHC sees two counterrotating beams of protons colliding head-on with each other every twenty-five nanoseconds (ns). Some sixteen hundred superconducting magnets encircle the two proton beams in a cryostat facility that maintains a temperature of 1.9 degrees Kelvin $\left(-271^{\circ} \mathrm{C}\right)$, which, as one of its posters proclaims, makes " $\mathrm{LHC}$ the coolest place on earth!”' After analyzing trillions of proton-to-proton collisions, on July 4, 2012, two independent teams of experimental physicists working on the LHC made the historic announcement of the discovery of the Higgs particle, which had been postulated way back in 1964 .

The event of this discovery elucidates several semiotic themes that are integral to scientific activity and yet appear in open conflict with some of its ideals of objectivity, materiality, and quantification. I would like to begin with the puzzling notion of a signature with which physicists carry out experimental searches for new particles that are not observed directly but may be deduced from traces left in the decay of high-energy collisions. While this is a preliminary statement, the concept of signature raises a fundamental and provocative question: How is a thing linked to a sign in a way that facilitates its possible discovery? In confronting this question, which devolves on the interlocution of the material and the mental, this article draws on medieval semiotics of Paracelsus, Böhme, and Weigel and tries to restore to its rightful place the extraordinary notion of signature, which has disappeared from the scholarly vision since the Enlightenment (Foucault 1970; Agamben 2009).

Second, the routine manner in which signatures are sought from decays of particle collisions hints at a schematic like language. By "language," here, I mean the technical and mathematical vocabulary used by physicists worldwide, which implies a collective, shared orientation and on the basis of which scientists lay

Alvarez-Gaume, for spiritedly setting up contacts in the field even as he stayed steadfast in his refusal to be easily impressed; James Wells, in explicating theories long after the lambent flame of fieldwork had waned; and Cesar Gomez, for languidly outlining an exquisite alchemy of analytic philosophy and physics. My greatest intellectual debt remains to my teacher Jit Singh Uberoi, who has single-handedly disclosed the pivots of modern European thought. But for his formidable and Promethean gift of knowledge, my life would have been nasty, brutish, and short. I thank the Wenner-Gren Foundation for their dissertation grant, which aided and enabled the timely completion of fieldwork at CERN.

1. The function of cryogenics, or cooling to low temperatures, is to keep the magnets steady in a superconducting state, i.e., with zero resistance. 
adroit claim to those currents of inspiration beyond reality or empirical circumstance (Giudice 2010). To be sure, it was somewhat expected that the LHC experiments would observe a Higgs particle in the mass range of 115 to 135 gigaelectron volts $(\mathrm{GeV})$, possibly decaying into lighter particles such as photons, B mesons, or Tau leptons, among others. But how the diphoton final state emerged as the cleanest experimental signature of the Higgs is inexplicable unless it is resolved through the lens of interpretation and meaning. This I propose to show on the basis of scrupulous ethnography. Perhaps one could even say that the denouement of the Higgs discovery, stirred through the diphoton signature across various distributions of background, may serve as a test case for scientific semiosis.

Finally, the problem of detecting and searching for uncharted possibilities of meaning among countless collisions is not an opaque event that happens by itself. Rather every fact of discovery entails truth claims that hint at the nature of judgment and convictions of a community. Here, what stands out in sharp pertinence is the open nature of debate, dissent, and disagreement among physicists. To insist on the experimental physics community's discussions as lending force and cohesion to the determination of physical reality is as controversial as it is significant (Pickering 1984; Knorr-Cetina 1999). Science is so accustomed to separating the subjective from the objective that it is incapable of appreciating even impersonal human presence, such as the constructive role of judgment, in showing something important about the physical world (Heisenberg 1958; Putnam 2002). On the other hand, I shall go into some detail to show how physicists' judgment is the framework in which all their investigations, including their controversies, squabbles, and doubts, are given shape and meaning. This should not be understood in unduly psychological or behavioral terms but rather as the logical limit by which any activity or thought acquires sense.

These questions - namely, the relation of thing and sign, their embedding in language, and the power of judgment to arbitrate reality - not only express internal features of experimental physics but also help reveal the full relevance of semiosis to the decoding of scientific consciousness. Drawing on two and a half years of ethnographic fieldwork at the LHC complex in Switzerland, this article probes the astonishing inventiveness that attaches to the covenant of signs and objects by which the pace of experimental physics is set. Much that I say here builds on my previous remarks on the signature of physics (Roy 2014). The chief novelty introduced here is a presentation of ontology, which is clearly and selfconsciously conceived along the lines of language, that is, viewed as a product of logic and reflection rather than of any immediate sensation. 
Lately in anthropology, there has been a great deal of discussion on the inevitability of ontology (Descola 2013), representations of reality (Keane 2013), obduracy of objects (Latour 2000), and so on. Yet "the ontological turn" (Kelly 2014), in my view, does not offer any radical methodological breakthrough or substantive canon of interpretation since it is inherently undialectical. To put it differently, the mediation that the ontological turn claims for materiality is paradoxically such that it can remain the same. On the other hand, this article strives to show that experimental physics is a game of constant flux interweaving at every moment language, thought, and materiality. That is why the methodology adopted here rejects at the outset any kind of repose on summa rerum. The emphasis instead is on locating the general form in which the dynamic and self-transcending character of materiality may be disclosed, thus moving away from the perennial question on the relation of representation to reality to the more promising question on the relation of reality to possibility (Wittgenstein 2001). How this may be achieved between two conflicting aspects of sciencethe spontaneity of discovery and the systematicity of method-is the focus of this article. But first a quick word on the nature of experiments conducted on the LHC.

The LHC is the current flagship project of the European Organization for Nuclear Research, or CERN. The accelerator lies in a seventeen-mile underground tunnel straddling the border of Switzerland and France. On the morning of November 30, 2009, when it successfully accelerated two counterrotating proton beams around the full seventeen miles at 1.18 teraelectron volts $(\mathrm{TeV})$ of energy, the LHC became the highest energy particle collider in the world. The two parallel proton beams are made to collide at four designated points where detectors have been placed to record the "data," or the product ensuing from the collisions. Detectors, in general, have four major components: (i) an inner tracker, which measures the momentum of charged particles; (ii) an electromagnetic calorimeter, which stops, absorbs, and measures the energy of light particles such as electrons and photons; (iii) a hadronic calorimeter, which samples the energy of hadrons, such as protons or neutrons, which interact via the strong nuclear force; and (iv) a muon tracker that measures the direction and momenta of energetic muons that pass through layers of dense material without interacting. The site of each detector forms a distinct experiment pursuing specific physics goals. The two large experiments, based on general-purpose detectors, are ATLAS and CMS, designed to investigate the widest range of physics discoveries, whereas LHCb and ALICE are specialized experiments that look closely into the area of flavor physics and heavy ions, respectively. 
Implicit in the design of the detectors, and the experiments by extension, is an overall semiotic element that from the outset is involved not just with indexicality, qualisigns, or abductive reasoning but also with the form necessary to the very conception of scientific knowledge, the intrinsic relation of sign and object. Let me explain. There is no doubt that features of particle behavior are sought on the basis of particular qualia - the momentum of a particle from the track of its curvature in the magnetic field or the strength of an interaction from the transverse size of a particle shower ${ }^{2}$ - that are used to generate a snapshot of what occurs at the moment of collision. That this is achieved through the use of specialized materials in the detectors is also worth mentioning. For instance, the use of silicon pixels in the ATLAS detector is done with the aim of picking up electric signals from colliding particles. Lead tungstate, which scintillates when electrons and photons pass through it - that is, produces light in proportion to particles' energy_-gives optimal energy resolution in the CMS detector. But though these qualitative features play a key role in guiding physicists to particle behavior, from a larger semiotic point of view this is of limited consequence. At most, one would get a phenomenological account that yields an inventory of discrete particle tracks and interactions.

The experimental tradition of physics, on the other hand, is dominated by the category of signature, which refers to patterns revealed by the collision and decay of particles. In that sense, it implies an organic conception and concerns the form or conditions under which particular qualia carries its power of representation. That it can do so is because, as I show, a signature expresses the ground of all relations from which assertions about any actual object can be made. Returning to the above example of particle showers, their recognition as qualia is not possible without knowing how the contribution of neutral particles, which do not leave a physical trace, can be converted into a detectable signature. ${ }^{3}$ Thus, the relations that a signature discloses are implicit by virtue of its form, which suggests that it is a logical rather than an empirical construct. In the final analysis, the thrust and intensity of signatures as engines of scientific discovery demonstrate that, notwithstanding the close engagement with the real, science freely rises to the formulation of the possible, which provides

2. The trajectory of high momentum particles is typically linear and in a direction transverse to the beam pipe. In contrast, particles with low momentum travel through in a crooked or spiral form in the longitudinal direction.

3. There is a stark difference in the interactions of charged particles, such as electrons and protons, and neutral particles, such as photons and neutrinos. While instances of the former can be founded on the evidence of the senses since these are physically discernible, the latter rely mostly on logic and computation. Any semiotic proposal, therefore, has to touch upon both these aspects, not separately, or combinatorially, but simultaneously. 
a more compelling basis to scrutinize those guarantees of materiality that it lays insistent claim to. Much in this condensed constellation remains to be hammered out. But the case for how dissemination of signs forms part of the logic of scientific discovery cannot be overstated, which returns one to the basic question, in what ways does a signature contribute to the understanding of nature?

\section{Signature and Nature}

Discoveries in particle physics are grounded in the theoretical framework of what is called the Standard Model, the celebrated paradigm of twentiethcentury physics. Aside from leaving gravity out, the Standard Model "covers everything else, from the reactions that fuel the sun to the forces that hold a snowflake together." 4 No direct contradiction to it has been found till date in any of the experiments. Nevertheless, there are some deeply unsettling, open questions that indicate that the Standard Model may not be the last word in particle physics. In particular, it lacks robustness at higher energies and is an "effective" theory only at low-energy scales; it makes no predictions on the masses of quarks and leptons; it does not explain the huge discrepancy in the strengths of the weak force and the gravitational force, and so forth (Giudice 2010; Wells 2012). These problems give a vigorous impetus to scientists to search for experimental deviations that could pave the way for a new paradigm. In other words, during experimental searches, it may be that physicists encounter something unusual in their data, which could be either $(a)$ consistent with Standard Model expectations, like the Higgs boson, or $(b)$ something totally unexpected and far removed from the Standard Model, such as "new physics."

I shall begin the discussion on signatures with the discovery of the Higgs boson. Now it was well known, both from theory and previous experiments, that a Higgs boson may not be directly observed because of its extremely short lifetime. Instead, its evidence has to be inferred from the manner in which it decays into lighter particles, such as $\mathrm{Z}$ bosons, muons, or photons. In other words, if a particle collision is successful in the production of a Higgs boson, it may leave its trace in the decay of photons or muons of a definite mass. Consider this with an analogy of, say, two cars involved in a collision. From the size of the tires left in the debris of collision, say, one may reconstruct what the original size, make, or model of the cars was. Likewise, from two discernible pho-

4. David Kestenbaum, "What Is Electroweak Symmetry Breaking Anyway?," FermiNews, January 23, 1998, available at https://www.fnal.gov/pub/ferminews/FermiNews98-01-23.pdf. 
tons or muons of a certain invariant mass or momentum found in the debris of collisions, physicists are led to the telltale signature of a parent Higgs particle.

However, this analogy — of reconstructing a parent particle from the debrishas to be taken with a pinch of salt. What needs to be underscored is that in relativistic quantum physics, decays are processes where particles, such as a Higgs, spontaneously transform into other particles, such as two photons or two muons. Here, decay is not a case of particles decomposing into constituent elements. New particles are spontaneously produced under the effect of field interactions in accordance with Einstein's famous equation $E=m c^{2}$. In other words, when fast-moving particles are collided with each other, some of their energy is converted into the creation of completely new particles. However, due to the relatively short-range quantum nature of interactions, physicists are not able to observe the new particles directly. Instead, their evidence has to be deduced from the decay products, and the term signature is used to characterize the decay products by which physicists are led to identify the source particles.

Does it not sound a little incongruous to approach the solemn realm of nature with the playfulness of signs? More importantly, how does this square with modern science's preoccupation with objectivity? Two and a half years of ethnographic research, based on intensive participant observation at CERN, leads me to affirm the opposition of fact and value (or thing and sign, or material and mental - however phrased) as a "total social fact" of scientific thought and conduct. Critics of science, who acknowledge the dynamics of this opposition, hasten to add that it is observed only in theory and more or less subverted in practice. ${ }^{5}$ Nevertheless, the provocation remains to find a concept in science that is executed on the strength of human judgment rather than any immutable fact "out there." The issue is capital and one must recognize the full weight of this opposition in order to appreciate the hypothesis I am arriving at: the deepest constituents of matter may not be conceived unless they have passed through the medium of signs.

Before the LHC began operation with two counter-rotating proton beams designed to collide at a record energy of $7 \mathrm{TeV}$, there were two particular channels of decay for the Higgs boson that physicists were most keen to focus on: (1) the decay into two $\mathrm{Z}$ particles that further decay to four leptons

5. The whole tenor of science studies in recent years has been to concentrate on scientific practice (Mol and Law 1994; Stengers 1997; Baird 2004). Latour argues, "Scientific practice is the only place where the object/subject distinction does not work" $(1999,123)$. Galison writes, "Increasingly, the idea of a pure science that finds application elsewhere does not fit the working practices of the laboratory" $(2008,117)$. This emphasis on practices somewhere raises the question, what happens to the reflex of critical scrutiny when it comes to theories and concepts? 
(Higgs $\left.\rightarrow Z Z Z^{*} \rightarrow \ell \ell \ell \ell\right) ;{ }^{6}$ and (2) the decay into two photons (Higgs $\rightarrow \gamma \gamma$ ). These two modes of decay allow for a clear detection of the Higgs particle and as a consequence are dubbed as "golden channels" in the particle physics community. When the actual collisions start taking place in March 2010, the diphoton decay channel turns out to be the more promising of the two and is swiftly extolled as the most robust signature of the long-sought-after Higgs boson.

The discussion of the diphoton-Higgs signature would not make much sense without bringing in the notion of "background." Background refers to identical and competing processes that often times fake a signal process. Now for every physics process there are two kinds of background, informants told me, that have to be taken care of: ( $a$ ) reducible background, which can be suppressed to some degree by making the technology of the detectors more sensitive and hermetic; and $(b)$ irreducible background, which is impossible to dislodge because it is, to a large extent, a by-product of actual physical interactions. The knowledge of what the background events of leading physics processes would be like is critical for experimentalists because the discovery potential of the signal depends on this knowledge. In the case of the diphoton signature, a huge, messy source of background happens to be photons produced by "bremsstrahlung," that is, braking radiation given off by accelerated particles when they are forced to travel in a curved trajectory, as in the circular accelerator LHC. Photons emitted from bremsstrahlung constitute the chief source of "irreducible background" (ATLAS 1994, 217).

The problem for experimentalists is to figure out, on a statistical basis, which photons are emerging as a consequence of bremsstrahlung, the irreducible background, and which ones are decaying from a possible Higgs source, the signal? I spoke to the convener of the Higgs search on the ATLAS experiment, Andreas Hoecker, and asked him how he deals with this form of background in his analysis. To my puzzlement, he replied rather nonchalantly that he did not believe in the concept of irreducible background. Now, Hoecker is a highly regarded figure in physics analysis, yet soon enough in the community I heard a few friendly murmurs of criticism of his approach to background: If he does not believe in the background, how can he discern a signal?

Skeptics were quick to suggest that Hoecker had previously worked on the electron-positron collider, BaBar, at SLAC, California, where the collisions are relatively clean as compared to a hadron collider, like the LHC, and was, there-

6. This is the notational form specifying the decay of a Higgs into two pairs of leptons mediated by a $Z$ boson and a virtual $\mathrm{Z}$ particle (with a mass less than $90 \mathrm{GeV}$ ). 
fore, prone to ignoring background contributions. ${ }^{7}$ At this point it was obvious that (1) there is continuity between experiments in the form of expertise that physicists bring with them from previous experiments and laboratories; (2) there flourishes a multiplicity of views on the extraction of signals in the subculture of experimental physics; and (3) there is a distinct identity to the work one does even in collaborations involving thousands of physicists in various capacities and positions. ${ }^{8}$

With some hesitation, I put to Hoecker the banter surrounding his approach to background in the ATLAS collaboration. He confidently replied that he was aware of the "conservative view in the community," but argued that no matter what, the topology of a signal event would always be different from the background. He then explained that while statistically plotting the decays, once a distinct peak starts emerging from the "invariant masses of the energetic photons," it would constitute the "signal that these are from a Higgs." On the other hand, photons from bremsstrahlung with differing masses, the irreducible background, would be all over the graph, falling in the "tail regions" of a Gaussian distribution. The overall shape of the distribution and the peak formed at $126 \mathrm{GeV}$ by the photons' invariant masses would, he said, constitute a conspicuous signature of the Higgs boson.

While conversing with Hoecker it was apparent that experimental physics illustrates a mode of thought and activity that reposes on the cusp of materiality and signs. In fact, without the mediation of the sign, there would be no discovery. Listening to the physicists on the way they extract signals from collision data, it became clear that the signal appears dormant unless it is summoned forth by a contrast. A case of a material discovery can only be settled after isolating the photons from bremsstrahlung (the background) against those issuing from a Higgs particle (the signal). This, however, is an act of discrimination that presupposes a subject, or more appropriately a community of subjects. The signal is real, and this is absolutely pivotal, but not because it is materially present in a collision. It is real because the physicist recognizes or receives it. Indeed, without the recognition of a physicist, there is nothing that answers to the notion of a signature.

The signature of physics shows that the task of probing matter is spread on a vast semiotic terrain where an object is conceived from the outset not as some-

7. Hadrons are composite particles and, therefore, generate a lot of debris, while electrons and positrons are elementary particles and lead to relatively clean collisions.

8. As stated on its website, the ATLAS experiment comprises about three thousand scientists representing thirty-eight countries, making it "a virtual United Nations"; seehttp://atlasexperiment.org/what_is_atlas .html\#2a. 
thing purely itself (a thing) but as something other than itself (a sign). To regard a pair of photons as a conclusive signal of a Higgs particle or high-energy muons as a signature of dark matter is a mode of recognition involving two elements - thing and sign - that are so completely taken up into each other that although admittedly they can be identified as different in reflection, they participate intrinsically together. One might think that this obvious recognition would have served as a privileged confirmation of the validity of semiosis for a physical science. Yet so far little attempt has been made to place the signature of physics at the center of scientific semiosis (Knorr-Cetina 1999). Indeed, it is significant that while researching the genealogy of this concept, the only appreciable references led me to medieval "doctrine of signatures" espoused with great rigor in the sixteenth century by Paracelsians.

In his treatise "Concerning the Signature of Natural Things," Paracelsus lays bare with outstanding flourish and ingenuity the vital currents of dynamically related occult sympathies, or signatures, encompassing nature from plants and minerals to spirits and stars. What makes signatures such powerful operators is the fact that at root all of nature forms a unity. His central argument, made in the context of medicine, is that the truth of a disease can be confirmed just by inspecting the symptoms, like rashes, boils, or fevers that express them, without appealing to anything external. Moreover, in a manner reminiscent of Plotinus's emanations, Paracelsus refuses to accept that in the impress and stimulus of symptoms or signatures there is anything occult or magical. He writes, "God did not create planets and stars with the intention that they should dominate man, but that they like, like other creatures, should obey and serve him. And although the higher stars do give the inclination, and, as it were, sign man and other earthly bodies for the manner of their birth, yet that power and that dominion are nothing, save only a predestined mandate and office, in which there is nothing occult or abstruse remaining, but the inner force and power is put forth through the external signs" $(1967,176)$.

This Hermetic conception of the signatura rerum, or the palimpsest of signs opening up new roads into the unknown, acquires intensity in the works of later Rhineland thinkers like Valentin Weigel and Jacob Böhme. For Weigel, the fundamental bond between man and god entails that not just spiritual knowledge, but even knowledge of nature cannot be received from the outside. "All vision depends in a decisive manner upon the eye itself . . . if it came from the object, we would all have the same perception" (Weeks 1993, 165). Böhme is even more emphatic in advocating that "without nature God is a mystery" $(1969,23)$ and that the unity of the godhead is expressed, reflected and included in each indi- 
vidual part of the universe, however limited or insignificant that may be (Weeks 1993). Only a mysticism alert to nature can speak of covert kinship and communicability of substances involving the part and the whole. Indeed, the reverence for nature, the discerning pulse of perception, the communion of macrocosm and microcosm render this approach radically distinct from any kind of popular or sentimental mysticism.

In Paracelsian Hermeticism, there lies a veiled cosmology whose feel we may have lost today. But from an anthropological perspective, it is of extreme interest to know if the sympathetic concourse of signs annunciating possible futures with prophetic force could be at work in science even today. Historically, of course, after the sixteenth century the doctrine of signatures steadily goes underground so that a hundred years later when Galileo attempts to read the book of nature, he no longer thinks it is written in the idiom of covert and qualitative signatures, but in unequivocal characters of integers and ellipses. (Dialectic had yielded to passive observation; cosmology had conceded to inert matter.) At the same time, the ardor and delight of invisible signs suffusing the universe remains undiminished and reminds us of both its antiquity and continuity. If the signature of physics deserves anthropological attention today, it is not for being mystical, but for once again being the semiotic framework that makes nature expressive and experiment the hunt for those expressive effects. I will now turn to the semiotic clarification of the signature from the standpoint of measurable effects as it unfolds and moves from the whole to the parts.

\section{Function, Form, and Transformation}

During two and a half years of fieldwork, it was repeatedly impressed upon me that the value of an experimental science consists not in summing up known empirical facts, but in its potentiality to call forth new facts. After all, the structure of matter is not simply to be described, but to be penetrated, probed and expounded (Giudice 2010). And the explanation of matter is not merely the realization of its existence as it is. Rather, the explanation consists in specifying the conditions under which it occurs-for example, how does matter arise in the early universe or how could extra dimensions of space-time be produced in the laboratory? In the foregoing, I have established that signatures are necessary for the detection of matter and are summoned forth by contrast, with background. Now the question arises, how does the evaluation of the strength of a signal take place? Let me address this issue by way of a controversy- the alleged discovery of leptoquarks. 
As mentioned earlier, all projections and deviations in experimental physics are measured by the compass of the Standard Model, which is a theoretical explanation of the interactions governing fundamental forces and elementary particles. However, as a possible extension beyond the Standard Model, and inspired by a certain symmetry between quark and lepton generations, the existence of leptoquarks has been proposed off and on by a number of theories. Two experiments, called $\mathrm{H} 1$ and ZEUS, on the electron-proton HERA collider at the Deutsches Elektronen-Synchrotron laboratory, in Hamburg, were keen on finding some deviation from the Standard Model. In November 1996, when the experimental run ended, scientists looked at the data and selected a sample of collision events with the aim of finding a scattered electron with the highest transverse momentum, known as "high pT electrons." The next step involved comparing the rate with Standard Model predictions and examining the outcome.

"The result for ten to fifteen of us, out of four hundred collaborators, took our breath away. There were too many interesting events. Really too many!" exclaimed Albert de Roeck, deputy spokesperson of CMS experiments, who was physics coordinator of the $\mathrm{H} 1$ experiment. He recalled that the $\mathrm{H} 1$ experiment had detected twelve events of high momentum electrons, whereas the Standard Model expectation gave somewhere between four and five events. The ZEUS experiment data showed that it had four events with a background of two, so it was a little less spectacular than H1. Two questions immediately came up: (a) How well was the background understood? and (b) Did the identified electrons show a resonant behavior in the electron-quark mass around a well-defined value? De Roeck remarked that the background looked under control, and the signal looked positively exciting. Could it be that the experiments had made a spectacular discovery?

The directorate of the German laboratory organized an official hearing with each of the experiment representatives separately (to prevent their respective views from getting influenced by each other). He appeared satisfied and asked for a joint paper to be submitted. A conference was even planned to make the historic announcement of this discovery. However, it gradually started sinking in that the $\mathrm{H} 1$ and ZEUS data did not exactly match, de Roeck narrated. They were off by 10 percent in the mass of the object spotted. That was a bad sign. In March 1997, the collider started operations again bringing renewed hope for fresh confirmation. "But there was no luck with more events," de Roeck said. A few months later it became evident to most experimentalists that the new 
data offered, "inadequate strength for a signal of 'new physics' and the discovery [of leptoquarks] was not claimed," de Roeck concluded.

My aim in outlining this controversy is to emphasize that the initial feeling of "vividness" to the signal $(E P)$, followed by doubt, that is finally supplanted by certainty forms a kind of system in science. And while the system is not set on ironclad rules, it is bound up with the evolution of debate and discussion (Pickering 1984). ${ }^{9}$ This has its methodological rationale. For while establishing the character of a signature is important, so is assessing its significance. However, to gauge this significance, there are no strict rules. Instead, physicists have to rely a great deal on tacit knowledge and craft skills. Armed with this tacit knowledge, experimentalists are called upon to play a leading role in validating discoveries. In borderline cases, it is not uncommon to observe a physics analyst claiming that a distribution shows "significant" excess of events outside of the Standard Model, while a group of colleagues radically disagree. The manifestation of significance is, as Peirce notes, exhibited at the level of pragmatics $(E P)$.

At every stage, statistical flukes and fluctuations - that is, errors in measurement - can affect the strength of a signal. Statistical techniques become indispensable in computing "whether a result might be less statistically significant than it looks more informally; but conversely, looking more informally is always necessary to tell you whether a result might be less reliable than statistical arguments might tell you." ${ }^{\prime 10}$ Experiments also contain a high degree of systemic uncertainties, connected with accelerator and detector design, which necessitate their own kind of sanity checks and cross-checks, including checks by eye (ATLAS 1994). After advancing through the threshold of errors, biases, and simulations, physicists then try to infer whether the data shows anything actually interesting or not, that is, if a discovery of a new particle or a phenomenon can be claimed. However, while the echoes that signals of discovery elicit may be visceral or practical, the accountability they seek is always logical. It is this entailment that lies at the heart of all critical and interpretive proceedings in experimental physics.

9. Like the agrarian calendar of the Kabyle peasantry, annual conferences, such as the International Conference on High-Energy Physics and the Rencontres de Moriond modulate the "real, practical time" (Bourdieu 1990) of the physics community. The anxiety to announce major findings at annual conferences also means that these witness the highest pitch of debates in the scientific calendar.

10. Matt Strassler, blog entry, July 8, 2012, available at https://profmattstrassler.com/articles-and-posts /the-higgs-particle/the-discovery-of-the-higgs/higgs-discovery-skill-and-a-little-luck/. 
By convention, the physics community considers the observation of a signature as valid if a significance of $5-\sigma$ standard deviations can be obtained. The $5-\sigma$ level is simply another way of expressing that a model or a theory has a 0.00003 percent chance of being false. There is a distinct sense in the experimental community that every result is a contingency, which may or may not have come into perceptible form. The feeling of contingency gets more aggravated in cases involving "new physics." That is why claims and counterclaims establishing a 5- $\sigma$ level for new physics discoveries are furiously debated and discussed. However, highlighting differences of opinion among scientists "does not force one to take a radically relativist stance toward experimental conclusions" (Galison 1987, 227). On the whole, I found that extraordinary caution and constraint is urged in claiming signals of discovery.

Projecting a statistical significance is only one phase in the process of establishing the robustness of a signal. There is another dimension that informants suggest contributes to the understanding of signal strengths, namely, the performance of the machine, which I will touch upon now. In experimental physics, ordinarily a distinction is observed between two core concepts, production cross section and luminosity. Production cross section simply means the probability of a certain process of interaction to occur during collisions. Luminosity stands for the measure of the number of collisions per unit of area (microbarn) per unit of time (second). Informants explained that the cross section is always at a given center-of-mass energy, that is, a fixed number dependent on specific physics processes, while luminosity is a factor controlled by the parameters of the machine and hence dependent on the state of technology.

In discussions with various informants, I gathered the distinct recognition that while physicists can measure the cross section of a given process, they cannot change it since, they claim, it is given by nature. Inversely, what falls under human control is the performance of the machine, which therefore can be consistently improved upon. The cross section is "fixed by nature and depends on the details of the interaction we wish to study. Only the luminosity, or number of collisions, is under our control. Since our analyses depend on gathering as large a sample as possible, we try to design an accelerator, which delivers as high a luminosity as we can."11 High machine luminosity can be achieved in a number of ways - for instance, by increasing the number of particles in the accelerator such as grouping particles into "bunches," by increasing the frequency at which collisions occur, by focusing the beams more tightly by apply-

11. Posted by Ted Kolberg on February 21, 2009, at http://blogs.uslhc.us/lhc-luminosity-and-energy. 
ing strong magnetic fields as they approach the interaction point where particles collide, and so on. It is this vital sense of technological efficiency, on which increased luminosity depends, that leads physicists to greater confidence in carrying out their experimental searches for Higgs and other particles.

In no sphere of knowledge is the contrast clearer between physical nature and human control; in none perhaps has there been so much of both. Predicated on this distinction, collisions and interactions take place and leave indelible traces on the experiments, which the detectors, duly prepared, can manage to capture and which are subsequently analyzed by scientists. That such vibrant traces should exist is entirely consonant with scientific rationale, but only after nature has made its mark, which leads to the reverence for such data and the affirmation of such methods as observation, quantification, measurement, and analysis (Uberoi 1984). It is noteworthy that human presence, by itself, is neither neglected nor considered insignificant; nothing is farther from science than a desire to be primitive (Rabinow 1996). Indeed, human agency in the shape of technology is part of its form to solicit and conquer the world.

The acknowledgment of human agency, however, confronts us with the question, how far does the province of technology reach the content of science? Surely, the human effort responsible for the performance of the accelerator is distinct from the human judgment that compels recognition of a meaningful signature? The answer to this question reposes on the basic distinction of function and form. The requirement of higher luminosity, which drives machine performance, is an exigent and functional aid to signal strengths, whereas the element of human judgment, which apprehends a signal amid countless collisions, is intrinsic and structural. As outlined above, without the perspective that recognizes in tracks of photons a signature of a Higgs boson, we cannot grasp how a collision provides the starting point of a new order of things in the precise moment of its decay. This perspectivization of nature, which the signature embodies, closes the gap of thing and sign in a single stroke.

The result is radical. For any classification of signs that devolves on the separation of the arbitrary and the conventional from the natural and the necessary, the signature of physics constitutes a disruptive presence. Sebeok (1999) recounts how every exposition of semiosis has endeavored to repudiate the "arbitrariness of signs," which Saussure made central to the study of semiology. Yet he bemoans the fact that the deeper we enter the realms of semiosis, the farther we recede from arriving at a satisfactory solution. For instance, the signs of classical antiquity, which come closest to natural signs like indices and signatures, operate on the mechanism of implication, $p \supset q$, but exclude the mo- 
dality of equivalence, $p \cong q$, that modern semiosis uses (Eco 1984; Manetti 1993). The cases of signature reported from the fields of extraterrestrial life (Helmreich 2006) and those of everyday life (Derrida 1982), seem singularly rooted in similitude and neglect to shed light on contiguity or part-whole relations. Even Peirce's $(E P)$ classification of icon, index, and symbol reposes on distinct modalities of firstness, secondness, and thirdness, or on combinations of similitude, causality, and convention. ${ }^{12}$

In my view, none of these proposals is persuasive. To think that a sign may be isolated, superposed with perspective, then energized with combinations of meanings as one goes along, is to admit that the ground of this activity is external and exigent. ${ }^{13}$ The remarkable advantage of the signature is that it discloses the (intrinsic) form by virtue of which the relation of sign and thing is possible. As Paracelsus had urged in his concept of signatures, do not look for something behind a phenomenon for it is itself the living manifestation of an order; that is, it reflects all the facts and relations of the universe (1967). The implication is monumental and twofold. First, the very nature of a signature is to be representative since the ground of its activity is driven by inner logic and propulsive necessity. That is why the seductive power of a signature is conspicuous in not what comes before it, like a theory, nor the facts that suggest it, or the processes that lead up to it. What is of consequence is what comes after the signal, how it may be assimilated to a theory, the uses that it can be put to, the facts that it will render significant.

A second outcome of no less import follows. The self-identity of a signature is not static but dynamic in the sense that it readily admits equivalence and exchange between units that are utterly different, like part and whole. The suggestive virtuosities of this exchange are best encapsulated in religious thought and cosmogony where the microcosm, by degrees, raises and surpasses its precariousness to express the multitudinous conditions of the macrocosm itself (Hubert and Mauss 1964; Durkheim 1965). Paracelsus accepts that genuine

12. Peirce was aware of the problems of his "trichotomy" for some indices were clearly symbolic and some of the mathematical symbols had strong iconic features (Liszka 1996; Short 2007). But the difficulty is that the remediation he seeks and proposes is always combinatorial (Parmentier 1994). Now, whether a combinatorial exercise is the only possible dialectical solution or should it constitute the touchstone of semiosis merits some reflection.

13. As a handy illustration of combinatorial reasoning, consider Peirce's formulation on "the First is that whose being is simply in itself, not referring to anything nor lying behind anything. The Second is that which is what it is by force of something to which it is second. The Third is that which is what it is owing to things between which it mediates and which it brings into relation to each other" (Peirce EP 1:248). This additive enumeration is at the root of another classification into qualisigns, sinsigns and legisigns (EP 2:291). 
communicability is addressed when each part is not itself but has the possibility of transforming and passing into the whole (Weeks 1993). The force and effect of this obscure phrasing is evident in every step of relativistic quantum physics. Consider how matter quarks get their masses from the Higgs field, which is postulated as the fountainhead of matter in the universe, or how a Higgs can transform to two $\mathrm{Z}$ particles, which further decay into charged leptons and antileptons, and so on. In other words, the syntax of any signature is defined not by abstract, static features, but through unceasing motion and transformation, the wonders of which are unflinchingly observed in the recesses of subnuclear matter.

The covenant between sign and thing has been richly scrutinized (Nöth 1990). Yet I think we have not adequately acknowledged the methodological differences that underpin, that envelope, the different trajectories of semiosis. ${ }^{14}$ Analytically, Peirce has shown us that the creative chain of semiosis is infinite. On the other hand, the relevant framework for signatures is not that of evolution, but of simultaneity. "Tout est conspirant," Leibniz proudly avers in the New Essays (1899). But the notion of sympathetic concomitance finds no demonstrable lodging or credence in secular, modern thought, whether of positivistic or hermeneutic persuasion (Cassirer 1957; Uberoi 1984). This is so profound and far-reaching that I can scarcely formulate it with requisite clarity. Uberoi eloquently calls the analytic paradigm of signatures as the "science of the symbol," which he contrasts with the modernist slant of the "science of the system" $(1984,73)$. It suffices to say that the science of the symbol speaks from the margin; it is decidedly of the occult and the underground. But it could well serve as the prototype of scientific semiosis. That move lies at the heart of this article.

\section{Measure of Reality}

The power and priority I have claimed for signatures has one final implication for semiosis that I wish to consider here in the last section. So far I have described the path taken by signatures, which makes every attempt to go beyond representation to engender and install material effects. Perhaps the strongest argument for the insistence that signs are necessary for the conception of things, and not just their interpretation, is occasioned by instances of unanticipated dis-

14. Once the underlying methodological orientations are grasped, it becomes clear how far apart Paracelsus and Peirce stand from each other or for that matter Leibniz and Saussure. 
covery. A whole new outlook opens up when reality is interwoven with possibility, such as in cases of speculative particles, where the signature passes into the limitlessness of play. Incidentally, this boundary condition is paramount for an experimental science.

In physics, a common signature called "missing transverse energy" (ET) fuels the search for new physics, or physics beyond the Standard Model. Missing transverse energy is simply energy, which may not be detected, but is required due to principles of conservation of energy or momentum. It constitutes a generic signature of physics beyond the expected. The upshot is momentous. Usually we labor under the assumption that there are things, which are revealed as signs. In the case of unanticipated discoveries made through missing ET, however, we have the reverse situation: a sign may stand for a thing that does not exist! Bizarre as it may seem at first glance, it neatly underscores how it is the power of possibilities that persuades scientists to look for signatures of things not yet experienced. Wittgenstein would find this immensely reassuring that futurity is not lacking in grammar (2001). Yet from the standpoint of anthropology, the issue raises an entrenched methodological question: What happens to ontology if signs do not have to proceed from reality (Keane 2003)?

I am not certain if I have a clear answer. But I will put forward from fieldwork a few comments on truth, which may help orient the discussion on ontology. Here Victor Turner's insight into different levels of symbolic meanings and how they may be deduced serves as a useful guide. Turner discerns a symbol's (1) exegetical meaning, obtained from questioning informants about observed ritual behavior, of which they are fully aware, (2) an operational meaning, which reveals its latent sense (of which the subjects are only marginally aware), and is derived from observing not only what is said, but also its usage, and (3) its positional meaning, which refers to its relationship with other symbols in the total ritual complex (1967, 50-52). The operational level of symbolic meaning drew my attention to an aspect of signatures that its manifest usage did not, which I advert to here.

During fieldwork I observed that discoveries and evidence were always debated against the standard of truth. It was quite a familiar sight at CERN to see talks organized with titles like "The Truth of the Top Quark" (February 14, 2008) and "How Charming is the Truth: Search for Neutral Currents" (July 1, 2008). Even at the conversational level, truth featured prominently. After a seminar on "CP Violation in B mesons," most members of the audience came out of the hall heatedly discussing how this could be true. When news came in from the Tevatron collider at Fermilab, in Illinois, on the exclusion of the Higgs mass in 
the window of 160 to $170 \mathrm{GeV}$, it created an outright commotion at CERN. A presentation given on the topic "Why I Never Believed the Tevatron Higgs Sensitivity Claims for Run2A and B" by Michael Dittmar on March 19, 2009, was particularly engaging. The discussion following the talk brought out perhaps the entire gamut of emotions that physicists are capable of-accusation (of fabricating the plots), defamation (of the Tevatron searches), erosion (of trust in scientific collegiality), and, above all, "Is it true?"

A cursory reading might suggest that in most informants' usage, the term truth coincides with reality. Entities, which are really out there, are true. But a sustained reflection, especially in relation to the work done on signatures, shatters any such simplistic notion of truth as a mere synonym or description of reality. For instance, originating in a common source, what permits physicists to distinguish photons decaying from a Higgs (a signal) from photons of bremsstrahlung (an illusion)? In the daily work with which experimental physicists are engaged, sifting signals from backgrounds, the veneration given to truth forces the didactic conclusion that truth is a yardstick, a criterion, to distinguish reality from illusion. No matter how routine and cliché, there is a palpable sense in which truth means much more than reality.

If I were to accept the performative utterances of truth as a description of reality, physicists' pursuits would be limited to the niche of reality. But the manner in which they carry out their appraisal of signatures makes it plain that while as physicists they are obviously concerned with physical reality, what they implicitly enact is the vivid notion of truth as a measure of reality. In short, reality is not given of which certain knowledge is thereby gained, but it is the certainty of knowledge as true, which constitutes the basis of their quest for physical reality. And the more one focuses on signatures in laying bare this quest, the more obvious it becomes that the thought of this community never ceases at the checkpoint of reality, but gropes beyond, toward the possibility of thoughts and things.

Currently in French anthropology, an emphasis on ontology is developing with pressing urgency (Kelly 2014). It is manifested perhaps nowhere as clearly as in the works of Bruno Latour. In a short essay, Latour reflects on the importance of materiality where his chief argument is: restore the rights of the object, recognize its ability to mobilize orders of existence, and reconfigure language and society. The result is "objectivity," which is "not a special quality of the mind, an inner state of justice and fairness" but simply the obduracy of objects, "how they object to what is told about them" $(2000,115)$. Latour recognizes the critical importance of language in the scaffolding of objects, but he is so capti- 
vated, dare one say obsessed, with materiality that he elects to place the entire accent on what he calls "the thingness of the thing."

The inquiry into signatures, like that of missing transverse energy or diphoton decay of the Higgs, shows that the value with which physicists endow a fact, such as a discovery, goes beyond the attribute of materiality into meaningfulness and possibility. It would be no hyperbole to suggest that scientists encrust remote possibilities through sign and meaning so vehemently that there is perhaps no analogous counterpart in poetry or art. What I find particularly perplexing in Latour's analysis is that if existence of an object alone were decisive, then science would have no category of "error." Error refers to the illusions of an object and constitutes the very sovereignty of the scientific (Cassirer 1957). At seminars and meetings, I heard over and over questions like, "How well have you estimated the errors from systematics?" "Are you basing your evidence solely against Monte Carlo simulations?" "To what degree can you claim a Standard Model Higgs from look-alike Higgs?” It is precisely because the object so marvelously fails them that one of the most enticing aspects of physics, namely debate and disagreement, acquires salience. Ultimately, if a signature of physics is stable it is not because it is a thing, but because it is immersed in a determinate language from which it cannot be drawn apart. Therefore, to attribute to objects the ability to generate abstract notions of objectivity or truth is mere shadowboxing and resurrects the myth that ontological guarantors can be found in the transparency of existence.

To conclude, the ontological turn toward materiality, agency, or existence is a simplification, which does not yield an adequate portrayal of the signs, errors or possibilities that saturate science. Or more pointedly, for it to do so, it must envisage foundations beyond the empirical. To this end, this article raises awareness on the remarkable concept of signature in physics, which as an act of meaningful apprehension rises above brute materiality and lends to contemporary science, almost imperceptibly, heretical possibilities first expressed in occult thought. In the luminous precedents of Hermetic thought, most notably in Paracelsus, one comes closest to discerning its analytical contours.

In the process, I have tried to clarify some of the methodological assumptions implicit in modern theories of semiosis and how the signature of subnuclear physics ensues in the breach of contract between things and signs. In the case of linguistic signs, the signifier and the signified share a fitful relation. This arbitrariness, as is well known, explains the generative potential and diversity of languages (Saussure 1983; Eco 1984). At the other extreme are discourses, which incline toward the sensuous character of things and inhabit the forecourt 
of ontology (Hacking 1999). The contrast in the two extreme positions is reflected in the division of the arts and the sciences (Nöth 1990). While the ultimate grounds of this classification remain enigmatic, the question I asked myself at the beginning of my research was can there be a thing, which is both itself and at the same time ineluctably turns into something other than itself, a sign?

Symptoms and signatures are impulses of a concentrated exchange where there exists a necessary relation between signifier and signified, which nonetheless submits to a language for the revelation of its true destiny. Texts in medical semiology attest to this alluring relation: rashes, boils, and fevers are not merely representative of pox, but emerge and disappear with the disease. Likewise, what the signature of physics discloses is that sign and thing are not extraneous juxtapositions, but inwardly linked and interlocked relations bound by the perspective of a subject, or more appropriately, the community of subjects. This expectation is confirmed when one recapitulates their various substantive merits, such as: signatures are not arbitrary signs but are formed by necessity; their ability to reach out to the possible is their inalienable possession; and their power of communicability and exchange rests not only on similitude but on difference and otherness as well. Without this many-sidedness that signatures embody, physics may never have disclosed its oracles on matter and energy.

From Gottfried Leibniz to René Thom, the question of how semiosis can yield new landscapes has held us in thrall. It is against this absorbing background that I have tried to analyze the assurance and finality that signatures bring to Bacon's famous simile of "nature being put to the question." However, from this investigation into signatures laden with meanings, given by a community and comprising a value, I do not conclude that science is personal or that physicists have a "feeling" for science (Fox-Keller 1983). On the contrary, what distinguishes the scientific subject from known feelings of subjectivity is the intellectual renunciation it exerts. Impartiality is not a negation of feeling, only an impersonal feeling. That is not a weak point. The signature of physics lays insistent claim to the subject, who is there to record a signal. And to make a record is to imprint a judgment. It is this very possibility that defines an experimental science.

\section{References}

Agamben, Giorgio. 2009. The Signature of All Things: On Method. New York: Zone.

ATLAS. 1994. "Techincal Proposal for a General-Purpose pp Experiment at the Large Hadron Collider at CERN.” Available at https://atlas.web.cern.ch/Atlas/TP/tp.html. 
Baird, Davis. 2004. Thing Knowledge: A Philosophy of Scientific Instruments. Berkeley: University of California Press.

Böhme, Jacob. 1969. The Signature of All Things and Other Writings. Cambridge: Clarke. Bourdieu, Pierre. 1990. The Logic of Practice. Stanford, CA: Stanford University Press.

Cassirer, Ernst. 1957. The Philosophy of Symbolic Forms. Vol 3. New Haven, CT: Yale University Press.

Derrida, Jacques. 1982. Margins of Philosophy. Chicago: University of Chicago Press.

Descola, Phillipe. 2013. Beyond Nature and Culture. Chicago: University of Chicago Press.

Durkheim, Émile. 1965. The Elementary Forms of the Religious Life. New York: Free Press.

Eco, Umberto. 1984. Semiotics and the Philosophy of Language. Bloomington: Indiana University Press.

Foucault, Michel. 1970. The Order of Things: An Archaeology of the Human Sciences. New York: Vintage Books.

Galison, Peter. 1987. How Experiments End. Chicago: University of Chicago Press.

- 2008. “Ten Problems in History and Philosophy of Science." Isis 99:111-24.

Giudice, Gian Francesco. 2010. A Zeptospace Odyssey: A Journey into the Physics of the LHC. Oxford: Oxford University Press.

Hacking, Ian. 1999. The Social Construction of What? Cambridge, MA: Harvard University Press.

Heisenberg, Werner. 1958. Physics and Philosophy: The Revolution in Modern Science. New York: Harper.

Helmreich, Stefan. 2006. “The Signature of Life: Designing the Astrobiological Imagination.” Grey Room 23 (3): 66-95.

Hubert, Henri, and Marcel Mauss. 1964. Sacrifice: Its Nature and Functions. Chicago: University of Chicago Press.

Keane, Webb. 2003. "Semiotics and the Social Analysis of Material Things." Lanquage and Communication 23 (3-4): 409-25.

- 2013. “Ontologies, Anthropologists, and Ethical Life." HAU: Journal of Ethnographic Theory 3 (1): 186-91

Keller, Evelyn Fox. 1983. A Feeling for the Organism: The Life and Work of Barbara Mcclintock. New York: Freeman.

Kelly, John D. 2014. "Introduction: The Ontological Turn in French Philosophical Anthropology." HAU: Journal of Ethnographic Theory 4 (1): 259-69.

Knorr-Cetina, Karin. 1999. Epistemic Cultures: How the Sciences Make Knowledge. Cambridge, MA: Harvard University Press.

Latour, Bruno. 1999. "For David Bloor . . . and Beyond: A Reply to David Bloor's AntiLatour." Studies in History and Philosophy of Science 30 (1): 113-29.

- 2000. "When Things Strike Back: A Possible Contribution of 'Science Studies' to the Social Sciences." British Journal of Sociology 51 (1): 10-23.

Leibniz, Gottfried Wilhelm. 1899. Nouveaux essais sur l'entendement humain. Paris: Delagrave.

Liszka, James J. 1996. A General Introduction to the Semiotic of Charles S. Peirce. Bloomington: Indiana University Press. 
Manetti, Giovanni. 1993. Theories of the Sign in Classical Antiquity. Bloomington: Indiana University Press.

Mol, Annemarie, and John Law. 1994. "Regions, Networks, and Fluids: Anaemia and Social Topology." Social Studies of Science 24:641-72.

Nöth, Winfried. 1990. Handbook of Semiotics. Bloomington: Indiana University Press.

Paracelsus. 1967. The Hermetic and Alchemical Writings of Aureolus Philippus Theophrastus Bombast, of Hohenheim, Called Paracelsus the Great. Trans. A. E. Waite. New Hyde Park, NY: University Books.

Parmentier, Richard J. 1994. Signs in Society: Studies in Semiotic Anthropology. Bloomington: Indiana University Press.

Peirce, Charles S. 1992. The Essential Peirce: Selected Philosophical Writings. 2 vols. Ed. the Peirce Edition Project. Bloomington: Indiana University Press [cited as EP].

Pickering, Andrew. 1984. Constructing Quarks: A Sociological History of Particle Physics. Chicago: University of Chicago Press.

Putnam, Hilary. 2002. The Collapse of the Fact/Value Dichotomy and Other Essays. Cambridge, MA: Harvard University Press.

Rabinow, Paul. 1996. Essays on the Anthropology of Reason. Princeton, NJ: Princeton University Press.

Roy, Arpita. 2014. "Ethnography and Theory of the Signature in Physics." Cultural Anthropology 29 (3): 479-502.

Saussure, Ferdinand de. 1983. Course in General Linguistics. London: Duckworth.

Sebeok, Thomas A. 1999. "The Sign Science and the Life Science." Applied Semiotics 3 (6/7): 386-93.

Short, T. L. 2007. Peirce's Theory of Signs. Cambridge: Cambridge University Press.

Stengers, Isabelle. 1997. Power and Invention: Situating Science. Minneapolis: University of Minnesota Press.

Turner, Victor W. 1967. The Forest of Symbols: Aspects of Ndembu Ritual. Ithaca, NY: Cornell University Press.

Uberoi, J. P. Singh. 1984. The Other Mind of Europe: Goethe as a Scientist. New Delhi: Oxford University Press.

Weeks, Andrew. 1993. German Mysticism from Hildegard of Bingen to Ludwig Wittgenstein. Albany, NY: SUNY Press.

Wells, James D. 2012. Effective Theories in Physics: From Planetary Orbits to Elementary Particle Masses. London: Springer.

Wittgenstein, Ludwig. 2001. Tractatus Logico-Philosophicus. Trans. David Pears and Brian McGuinness. London: Routledge. 